\title{
Hydrogen sulfide upregulates heme oxygenase-1 expression in rats with volume overload-induced heart failure
}

\author{
CHAO-YING ZHANG ${ }^{1}$, XIAO-HUI LI ${ }^{1}$, TING ZHANG ${ }^{2}$, JIN FU $^{3}$ and XIAO-DAI CUI ${ }^{3}$ \\ ${ }^{1}$ Department of Cardiovascular Diseases, Children's Hospital Affiliated to the Capital Institute of Pediatrics; \\ ${ }^{2}$ Central Laboratory of Infection and Immunity, Capital Institute of Pediatrics; ${ }^{3}$ Central Laboratory for Clinical Research, \\ Children's Hospital Affiliated to the Capital Institute of Pediatrics, ChaoYang, Beijing 100020, P.R. China
}

Received January 4, 2013; Accepted February 25, 2013

DOI: $10.3892 /$ br.2013.87

\begin{abstract}
The present study investigated the role of hydrogen sulfide $\left(\mathrm{H}_{2} \mathrm{~S}\right)$, a novel gaseous transmitter, in chronic heart failure $(\mathrm{CHF})$ induced by left-to-right shunt, leading to volume overload. Thirty male Sprague-Dawley rats were randomly divided into four groups: the shunt group, the sham group, the shunt + sodium hydrosulfide (NaHS) group and the sham + NaHS group. CHF was induced in the rats by abdominal aorta-inferior vena cava shunt operation. Rats in the shunt $+\mathrm{NaHS}$ and sham $+\mathrm{NaHS}$ groups were injected intraperitoneally with $\mathrm{NaHS}\left(\mathrm{H}_{2} \mathrm{~S}\right.$ donor). Haemodynamic parameters were measured 8 weeks after surgery. In addition, left ventricular heme oxygenase (HO)-1 mRNA expression was measured by real-time PCR. Protein expression of HO-1 was evaluated by western blot analysis. Eight weeks after surgery, compared to the sham group, the left ventricular systolic pressure (LVSP) and left ventricular peak rate of contraction and relaxation $(\mathrm{LV} \pm \mathrm{dp} / \mathrm{dtmax})$ were significantly reduced; the left ventricular end-diastolic pressure (LVEDP) was significantly increased in the shunt group (all $\mathrm{P}<0.05)$. However, NaHS increased LVSP and LV \pm dp/dtmax $($ all $\mathrm{P}<0.05)$ and decreased LVEDP $(\mathrm{P}<0.05)$. Protein expression of HO-1 was significantly decreased in the shunt group compared to that in the sham group $(\mathrm{P}<0.05)$. NaHS increased protein expression of $\mathrm{HO}-1$ compared to that in the shunt group $(\mathrm{P}<0.05)$. HO-1 mRNA expression was significantly increased in the shunt $+\mathrm{NaHS}$ group compared to that in the shunt group $(\mathrm{P}<0.01)$. The present study demonstrated that $\mathrm{H}_{2} \mathrm{~S}$ may play a protective role in volume overload-induced CHF by upregulating protein and mRNA expression of HO-1.
\end{abstract}

Correspondence to: Professor Xiao-hui Li, Department of Cardiovascular Diseases, Children's Hospital Affiliated to the Capital Institute of Pediatrics, 2 Ya-Bao Road, ChaoYang, Beijing 100020, P.R. China

E-mail: 1xhmaggie@126.com

Key words: hydrogen sulfide, heme oxygenase-1, volume overload

\section{Introduction}

Congenital heart disease (CHD) is the most type of common cardiovascular disease of childhood. Left-to-right shunt CHD results in an increase in cardiac volume load. Sustained volume overload induces cardiac hypertrophy and ventricular remodeling, eventually leading to decreased cardiac function, which results in chronic heart failure (CHF); however, the pathogenesis of CHF has not been fully elucidated. Hydrogen sulfide $\left(\mathrm{H}_{2} \mathrm{~S}\right)$ affects a wide range of physiological and pathological processes in the cardiovascular system (1). $\mathrm{H}_{2} \mathrm{~S}$ plays an important role in the prevention of the development and occurrence of coronary heart disease and the protection against ischemic myocardial injury (2-4). Exogenous $\mathrm{H}_{2} \mathrm{~S}$ opens $\mathrm{K}_{\text {ATP }}$ channels to reduce myocardial infarct size $(5) . \mathrm{H}_{2} \mathrm{~S}$ exerts a protective effect on ischemic myocardium by inhibiting vascular endothelial cell apoptosis and promoting the regeneration of endothelial cells (6). In a previous study (7), we reported that increased myocardial collagen content (particularly type I collagen) in rats with volume overload caused CHF and treatment with sodium hydrosulfide (NaHS), an exogenous $\mathrm{H}_{2} \mathrm{~S}$ donor, resulted in a decrease of myocardial collagen content (particularly type I collagen) in the left-to-right shunt operation group. This suggested that $\mathrm{H}_{2} \mathrm{~S}$ plays a protective role in volume overload-induced ventricular remodeling. However, the mechanism underlying these changes has not been fully elucidated. Carbon monoxide (CO) is another important endogenous signaling molecule. Mammalian tissues continually produce $\mathrm{CO}$ as a result of the breakdown of heme by heme oxygenase ( $\mathrm{HO})$. $\mathrm{HO}$ degrades the pro-oxidant heme to $\mathrm{CO}$, biliverdin and ferrous iron. $\mathrm{HO}$ has been reported to exist as its isoenzyme forms, HO-1, -2 and -3. HO-3 is inactive and is not expressed in humans. HO-1 is expressed ubiquitously at low levels and its expression is rapidly induced by heme as well as other stresses, including hypoxia, hyperthermia, metals, oxidized low-density lipoprotein and inflammatory cytokines. By contrast, HO-2 is constitutively expressed and widely distributed in the body, with higher concentrations in the brain and testis (8). HO-1 is upregulated by a host of oxidative stress stimuli in the cardiovascular system (9). The HO-1/CO system is beneficial in the prevention of atherosclerotic lesion formation, protection of ischemic myocardial injury and regulation of blood pressure (10-15). Considering these findings, the 
issues that should be addressed include whether $\mathrm{H}_{2} \mathrm{~S}$ affects the $\mathrm{HO}-1 / \mathrm{CO}$ system and whether the interaction between $\mathrm{H}_{2} \mathrm{~S}$ and the $\mathrm{HO}-1 / \mathrm{CO}$ system is involved in the regulation of volume overload-induced heart failure. The present study was designed in order to elucidate these issues by investigating the expression of HO-1 in rats with left-to-right shunt and in shunted rats treated with NaHS.

\section{Materials and methods}

Animal model of left-to-right shunt. Experiments were conducted in accordance with the Guide to the Care and Use of Experimental Animals issued by the Ministry of Health, the People's Republic of China. Male Sprague-Dawley rats were provided by the Animal Research Centre of Peking University First Hospital. The rats were housed in plastic cages in a room with a controlled humidity of $40 \%$, a temperature of $22^{\circ} \mathrm{C}$ and a 12-h light cycle from 6:00 a.m. to 6:00 p.m. The rat model was established by an abdominal aorta-inferior vena cava shunt operation, as previously described by Ocampo et al (16). Briefly, 30 male Sprague-Dawley rats, weighing 120-140 g, were randomly divided into four groups: the shunt group $(n=8)$, the shunt + NaHS group $(n=8)$, the sham group $(n=8)$ and the sham + NaHS group $(n=6)$. Rats in the shunt and shunt + NaHS groups were anesthetized with $0.25 \%$ pentobarbital sodium (40 mg/kg, intraperitoneal injection). The abdominal aorta and inferior vena cava were exposed and a bulldog vascular clamp was placed across the aorta, caudal to the left renal artery. The aorta was punctured at the union of the segment, two-thirds caudal to the renal artery and one-third cephalic to the aortic bifurcation, with an 18-gauge disposable needle. The needle was slowly withdrawn and a 9-0 silk thread was used to suture the puncture of the abdominal aortic wall. In the sham and sham + NaHS groups, rats underwent the same experimental protocol as mentioned above, except for the shunt procedure. Rats in the shunt + NaHS and sham + NaHS groups were injected intraperitoneally with $\mathrm{NaHS}\left(\mathrm{H}_{2} \mathrm{~S}\right.$ donor) at $56 \mu \mathrm{mol} / \mathrm{kg} /$ day for 8 weeks, as previously described (17), whereas rats in the shunt and sham groups were injected with the same volume of normal saline (NS).

Measurement of haemodynamic parameters. At 8 weeks after the operation, rats in each group were anesthetized with $25 \%$ urethane $(0.5 \mathrm{ml} / 100 \mathrm{~g}$, intraperitoneal injection). After anesthesia, a cannula with a heparinized PP10 in PP50 catheter was inserted into the left ventricle (LV) through the right common carotid artery. The catheter was connected to a pressure transducer. The pressure transducer was connected to a data recording system (BL-420F, BioData Acquisition \& Analysis System; TME Technology Co., Ltd., Chengdu, China). The haemodynamic parameters, such as left ventricular systolic pressure (LVSP), left ventricular end-diastolic pressure (LVEDP), left ventricular peak rate of contraction $(\mathrm{LV}+\mathrm{dp} / \mathrm{dtmax})$ and left ventricular peak rate of relaxation (LV-dp/dtmax), were measured as previously described (18).

RNA extraction and cDNA synthesis. Total RNA was isolated from frozen LV tissue using the Total RNA Extraction kit (Tiangen Biotech, Co., Ltd., Beijing China; code no. DP419) according to the manufacturer's protocol and quantified
Table I. Primers used in the study.

\begin{tabular}{ll}
\hline Gene & \multicolumn{1}{c}{ Nucleotide sequence } \\
\hline HO-1 & \\
Sense & 5'-AGA GTT TCC GCC TCC AAC CA-3' \\
Antisense & 5'-CGG GAC TGG GCT AGT TCA GG-3' \\
GAPDH & \\
Sense & 5'-CAA GGT CAT CCA TGA CAA CTT TG-3' \\
Antisense & 5'-GGG CCA TCC ACA GTC TTC TG-3' \\
\hline
\end{tabular}

HO-1, heme oxygenase-1; GAPDH, glyceraldehyde-3-phosphate dehydrogenase.

by measuring the absorbance at $260 \mathrm{~nm}$. The quality of the isolated RNA was determined by measuring the 260:280 ratio. Subsequently, first-strand cDNA was synthesized using the First-Strand cDNA Synthesis kit (Tiangen Biotech, Co., Ltd.; code no. KR104) according to the manufacturer's protocol.

Relative gene expression analysis by real-time PCR. PCR primers were designed using commercial software (Beacon Designer; Bio-Rad Laboratories, Hercules, CA, USA) to produce an amplicon 75-150 bp in length. Primers used in the PCR assays are presented in Table I. Real-time PCR was performed with the ABI Prism 7500 system (Applied Biosystems, Carlsbad, CA, USA), using the Ultra SYBR-Green PCR kit (Beijing CoWin Bioscience Co., Ltd., Beijing, China; code no. CW0956). The thermal cycling conditions included an initial denaturation step at $95^{\circ} \mathrm{C}$ for $10 \mathrm{~min}$, followed by 40 cycles at $95^{\circ} \mathrm{C}$ for $15 \mathrm{sec}$ and at $60^{\circ} \mathrm{C}$ for $60 \mathrm{sec}$. A melting curve was determined at the end of each cycle to confirm the specificity of the primers and the purity of the PCR product. Results were analyzed using Applied Biosystems 7500 Real-Time PCR System Sequence Detection Software version 1.4 (Applied Biosystems) to obtain $\mathrm{C}_{\mathrm{T}}$ values (threshold cycles at which a statistically significant increase in detection of SYBR-Green emission intensity occurs). $\mathrm{C}_{\mathrm{T}}$ values were then normalized to a glyceraldehyde-3-phosphate dehydrogenase (GAPDH) endogenous control to account for variability in RNA concentrations between samples to obtain $\Delta \mathrm{C}_{\mathrm{T}}$ values. To obtain $\Delta \Delta \mathrm{C}_{\mathrm{T}}$ values, we subtracted the $\Delta \mathrm{C}_{\mathrm{T}}$ value for the control samples from that for the treated samples. The relative quantification value was then calculated as $2^{-\Delta \Delta \mathrm{C}_{\mathrm{T}}}$.

Western immunoblot analysis. LV tissue was lysed in a lysis buffer $[0.2 \mathrm{ml} 1 \mathrm{M}$ Tris-HCl (pH 8.0), $0.3 \mathrm{ml} 5 \mathrm{M} \mathrm{NaCl}$, $10 \mu 1500 \mathrm{mM}$ ethylenediaminetetraacetic acid, $0.1 \mathrm{ml} 100 \mathrm{mM}$ phenylmethanesulfonyl fluoride and $10 \mu \mathrm{l}$ Triton X-100, with water added to $10 \mathrm{ml}$. The extracts were clarified by centrifugation at $13,400 \mathrm{x} \mathrm{g}$ for $15 \mathrm{~min}$ at $4^{\circ} \mathrm{C}$. Protein concentrations were determined with the $\mathrm{BCA}^{\mathrm{TM}}$ Protein Assay kit (Thermo Fisher Scientific Inc., Waltham, MA, USA). Equal amounts of total protein $(60 \mu \mathrm{g})$ were resolved by sodium dodecyl sulfate-polyacrylamide gel electrophoresis (SDS-PAGE) on a $15 \%$ gel and transferred onto a polyvinylidene difluoride membrane (GE Healthcare UK Ltd., Little Chalfont, UK). The 
Table II. Heme oxygenase- 1 mRNA expression in the left ventricle $\left(2^{-\Delta \Delta C_{T}}\right)^{a}$.

\begin{tabular}{lcccc}
\hline & Shunt & Sham & Shunt + NaHS & Sham + NaHS \\
\hline No. & 8 & 8 & 8 & 6 \\
HO- & $1.86 \pm 0.29$ & $2.05 \pm 0.24$ & $5.86 \pm 0.61^{\text {b }}$ & $2.94 \pm 0.63$ \\
\hline
\end{tabular}

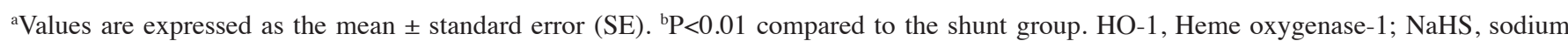
hydrosulfide.

membrane was blocked with $5 \%(\mathrm{w} / \mathrm{v})$ fat-free milk in TBST $[0.05 \%(\mathrm{v} / \mathrm{v})$ Tween in TBST] at room temperature for $1 \mathrm{~h}$ and then probed with rabbit polyclonal antibodies (Abcam Inc., Cambridge, UK) against HO-1 at a 1:500 dilution overnight at $4^{\circ} \mathrm{C}$. The membrane was then probed with horseradish peroxidase-conjugated goat anti-rabbit IgG (1:2,500; Santa Cruz Biotechnology Inc., Santa Cruz, CA, USA) for $2 \mathrm{~h}$ at room temperature. The reactions were developed with enhanced chemiluminescence reagents (Beijing TransGen Biotech Co., Ltd., Beijing, China) and the images were obtained by exposure to X-ray films (Kodak Life Science Imaging film, USA). The films were digitized with Bio-Rad Gel Doc XR (Bio-Rad Laboratories) and quantified with Quantity One software (Bio-Rad Laboratories). GAPDH blots were used as a loading control. The bands were normalized to GAPDH controls.

Statistical analysis. Data are expressed as the means \pm standard error (SE) and were analyzed by SPSS software, version 16.0 (SPSS Inc., Chicago, IL, USA). For the homogeneity of variance values, a comparison among groups was performed with one-way analysis of variance (ANOVA), followed by the Least Significance Difference test. For some heterogeneity of variance values, comparison among groups was performed with one-way ANOVA followed by Tamhane's T2 test. $\mathrm{P}<0.05$ was considered to indicate a statistically significant difference.

\section{Results}

LV haemodynamic parameters. Eight weeks after the operation, the shunt group exhibited significantly increased LVEDP $(24 \pm 7$ vs. $14 \pm 5 \mathrm{mmHg} ; 1 \mathrm{mmHg}=0.133 \mathrm{kPa}$, $\mathrm{P}<0.05)$ and significantly decreased LVSP and LV \pm dp/dtmax $(101 \pm 19$ vs. $145 \pm 26 \mathrm{mmHg} ; 3768 \pm 321$ vs. $5768 \pm 432 \mathrm{mmHg}$ / sec; $3219 \pm 219$ vs. $5312 \pm 418 \mathrm{mmHg} / \mathrm{sec}$, all $\mathrm{P}<0.05$ ), compared to the sham group. In addition, the shunt $+\mathrm{NaHS}$ group exhibited significantly decreased LVEDP $(19 \pm 6$ vs. $24 \pm 7 \mathrm{mmHg}$, $\mathrm{P}<0.05)$ and significantly increased LVSP and LV \pm dp/dtmax $(121 \pm 16$ vs. $101 \pm 19 \mathrm{mmHg} ; 4865 \pm 254$ vs. $3768 \pm 321 \mathrm{mmHg} /$ sec; $4138 \pm 207$ vs. $3219 \pm 219 \mathrm{mmHg} / \mathrm{sec}$, all $\mathrm{P}<0.05)$, compared to the shunt group. There was no significant difference between the sham and the sham + NaHS groups (Fig. 1).

HO-1 mRNA expression in the LV. Eight weeks after the operation, HO-1 mRNA expression tended to decrease in the shunt group compared to that in the sham operation group $(\mathrm{P}>0.05)$. The shunt + NaHS group exhibited significantly increased HO-1 mRNA expression compared to that in the shunt group

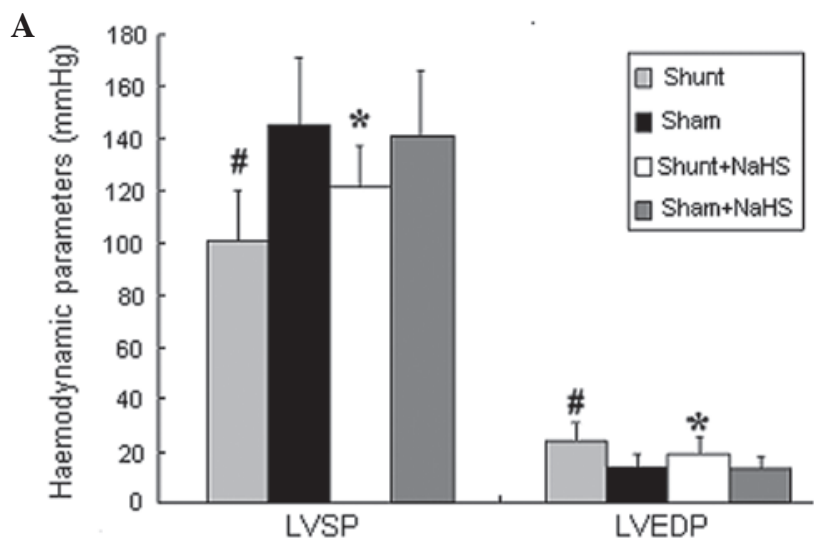

B

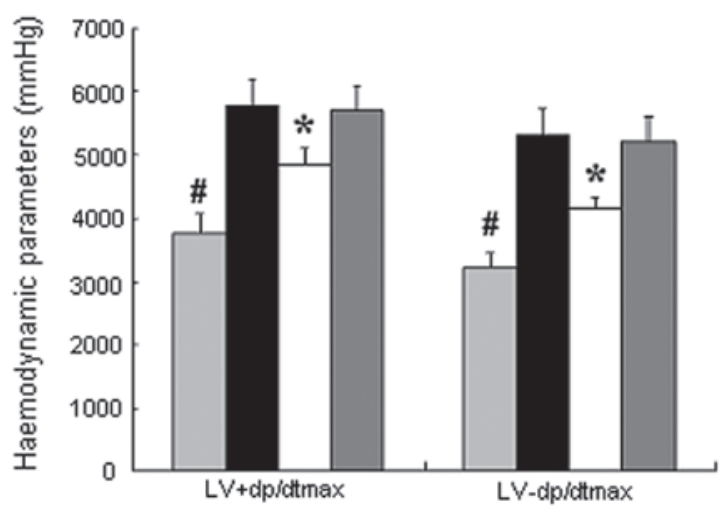

Figure 1. Haemodynamic parameters of rats in the four groups. Values are expressed as means \pm standard deviation (SD). ${ }^{~} \mathrm{P}<0.05$ vs. the sham group, ${ }^{*} \mathrm{P}<0.05$ vs. the shunt group. LVSP, left ventricular systolic pressure; LVEDP, left ventricular end-diastolic pressure; LV \pm dp/dtmax, left ventricular peak rate of contraction and relaxation; NaHS, sodium hydrosulfide.

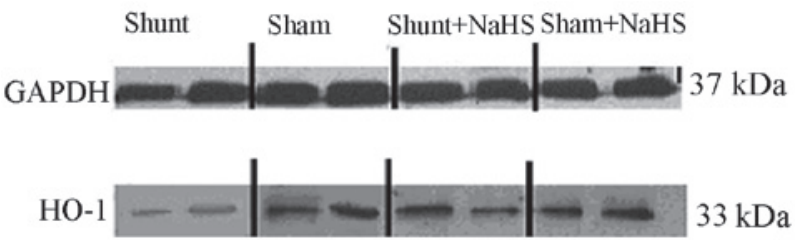

Figure 2. Representative western immunoblots for heme oxygenase-1 (HO-1) in left ventricular (LV) myocardial extracts. SDS-PAGE analysis shows HO-1 protein levels in the LV of rats. GAPDH, glyceraldehyde-3-phosphate dehydrogenase; NaHS, sodium hydrosulfide.

$(\mathrm{P}<0.01)$. There was no significant difference in HO-1 mRNA expression between the sham and the sham + NaHS groups (Table II). 


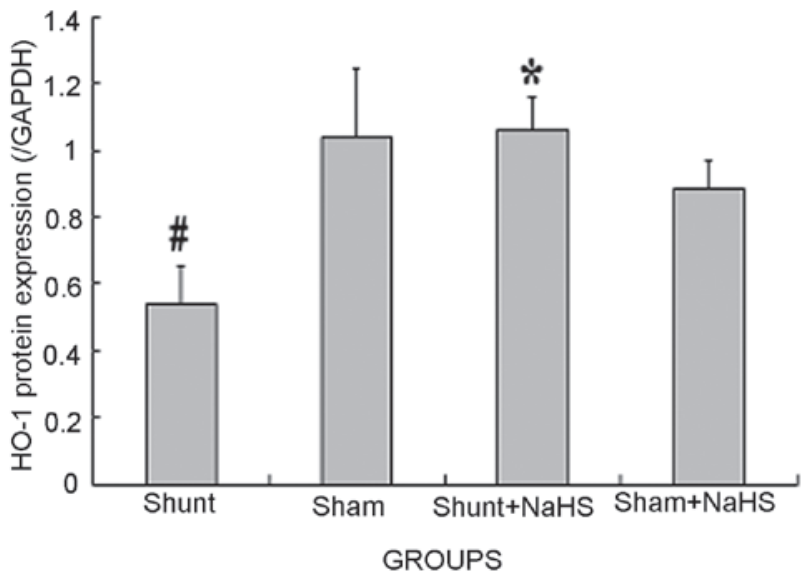

Figure 3. Cardiac protein expression bar graph in the left ventricle (LV) using the western blot analysis method, demonstrating the change in HO-1 protein expression. Values are expressed as means \pm standard error (SE). ${ }^{\#} \mathrm{P}<0.05$ vs. the sham group, " $\mathrm{P}<0.05$ vs. the shunt group. HO-1, heme oxygenase-1; GAPDH, glyceraldehyde-3-phosphate dehydrogenase; NaHS, sodium hydrosulfide.

Western blot analysis results of $\mathrm{HO}-1$ in the LV. Eight weeks after the operation, HO-1 protein expression was significantly decreased in the shunt group compared to that in the sham operation group $(0.54 \pm 0.11$ vs. $1.04 \pm 0.20, \mathrm{P}<0.05)$. HO-1 protein expression was significantly increased in the shunt $+\mathrm{NaHS}$ group compared to that in the shunt group $(1.06 \pm 0.10$ vs. $0.54 \pm 0.11, \mathrm{P}<0.05)$. There was no significant difference in HO-1 protein expression between the sham and the sham + NaHS groups (Figs. 2 and 3).

\section{Discussion}

CHD is one of the most common human birth defects, with an incidence of 6-8/1,000 live births (19). CHF is a common complication of left-to-right shunt $\mathrm{CHD}$ and ventricular remodeling is an important pathophysiological mechanism underlying volume overload-induced $\mathrm{CHF}$. With ventricular structural remodeling, cardiac function is compromised, which results in irreversible heart failure. Therefore, it is critical to elucidate the mechanism behind volume overload-induced $\mathrm{CHF}$ and perform timely interventions.

Similar to nitric oxide (NO) and $\mathrm{CO}$, which are considered as gaseous transmitters, $\mathrm{H}_{2} \mathrm{~S}$ has also been shown to be a gaseous transmitter which plays an important role in normal physiological processes as well as in the process/progression of several diseases. The four most important mammalian enzymes involved in $\mathrm{H}_{2} \mathrm{~S}$ synthesis are cystathionine- $\beta$-synthase (CBS), cystathionine- $\gamma$-lyase (cystathionase, CSE) and cysteine aminotransferase (CAT) in conjunction with 3-mercaptopyruvate sulfurtransferase (3-MST) (1). $\mathrm{H}_{2} \mathrm{~S}$ is synthesized from the sulfur-containing amino acid L-cysteine by either CBS or CSE, with pyridoxal 5'-phosphate used as a cofactor. Along with CAT, 3-MST produces $\mathrm{H}_{2} \mathrm{~S}$ using L-cysteine and $\alpha$-ketoglutarate as substrates. Both enzymes contribute to $\mathrm{H}_{2} \mathrm{~S}$ formation in the brain and vascular endothelium. Recent experimental studies demonstrated that endogenous $\mathrm{H}_{2} \mathrm{~S}$ synthesis was lower in hearts of an arteriovenous fistula-induced $\mathrm{CHF}$ model (1). In the present study, 8 weeks after the shunt surgery, there were significant changes in LVSP, LVEDP and LV $\pm d p /$ dtmax, indicating that $\mathrm{H}_{2} \mathrm{~S}$ may improve cardiac function in volume overload-induced CHF. Furthermore, the results indicated that long-term treatment with NaHS may not affect the left ventricular haemodynamic parameters in sham-operated rats, although it exerted a significant pharmacological effect under pathological conditions (such as CHF). This finding indicated that $\mathrm{H}_{2} \mathrm{~S}$ may protect the heart against CHF; however, its mechanism has not yet been elucidated. Endogenous $\mathrm{H}_{2} \mathrm{~S}$ is produced through the metabolism of terminal waste of sulfur-containing amino acids in the body. $\mathrm{H}_{2} \mathrm{~S}$ may occur as gaseous $\mathrm{H}_{2} \mathrm{~S}$ or NaHS. NaHS in the body may dissociate to sodium ions $\left(\mathrm{Na}^{+}\right)$and sulfur hydrogen ions (HS-). HS combines with internal hydrogen ions $\left(\mathrm{H}^{+}\right)$to generate $\mathrm{H}_{2} \mathrm{~S}$. Therefore, $\mathrm{H}_{2} \mathrm{~S}$ and NaHS are in a type of dynamic balance. NaHS may ensure the stability of $\mathrm{H}_{2} \mathrm{~S}$ concentrations in solution and the majority of intervention experiments on $\mathrm{H}_{2} \mathrm{~S}$ used NaHS solution (17). Therefore, in this study, we used NaHS solution as the $\mathrm{H}_{2} \mathrm{~S}$ donor.

$\mathrm{CO}$ is an endogenously derived gas formed from the breakdown of heme by the HO enzyme. Although long considered an insignificant and potentially toxic waste product of heme catabolism, $\mathrm{CO}$ is now recognized as an important signaling molecule that regulates numerous cardiovascular functions. Of note, alterations in $\mathrm{CO}$ synthesis are associated with several cardiovascular disorders, including atherosclerosis, septic shock, hypertension, metabolic syndrome and ischemia-reperfusion injury; restoration of physiological $\mathrm{CO}$ levels exerts a beneficial effect on several of these conditions, suggesting a crucial role for $\mathrm{CO}$ in the maintenance of cardiovascular homeostasis. $\mathrm{CO}$ causes relaxation of numerous vascular tissues and regulates blood pressure by activating soluble guanylate cyclase in vascular smooth muscle, leading to the production of cyclic guanine monophosphate (20). Considerable evidence supports a protective role for the $\mathrm{HO}-1 / \mathrm{CO}$ system against coronary artery ischemia-reperfusion injury. Pharmacological induction of HO-1 expression significantly reduces infarct size and the incidence of reperfusion arrhythmia following myocardial ischemia-reperfusion, whereas cardiac tissue damage is exacerbated by HO inhibitors (21-25). The induction of HO-1 may also have therapeutic benefits during $\mathrm{CHF}$. Upregulation of HO-1 expression during heart failure serves to mitigate pathological LV remodeling and reduce myocardial hypertrophy, oxidative stress and inflammatory activation. HO-1 overexpression promotes neovascularization and ameliorates apoptosis in the heart failure model (26).

$\mathrm{H}_{2} \mathrm{~S}$ and $\mathrm{CO}$ are involved in the regulation of several physiological as well as pathological processes. They have similar biological functions, as well as competitive and antagonist actions. For example, $\mathrm{H}_{2} \mathrm{~S}$ administered to rats with experimentally induced hypoxic pulmonary hypertension leads to an increase in plasma $\mathrm{CO}$ concentrations and an increase in pulmonary artery expression of HO-1 protein and mRNA (27). In the present study, 8 weeks after the shunt surgery, the expression of LV HO-1 mRNA and protein was significantly increased in the shunt + NaHS group compared to those in the shunt group. No difference in HO-1 expression was observed between the sham group and the sham + NaHS group. These findings demonstrated that $\mathrm{H}_{2} \mathrm{~S}$ may play a protective role in volume overload-induced heart failure by upregulating protein and mRNA expression of HO-1. 
The regulatory effect of NaHS on the HO-1/CO system pathway has not been fully elucidated. A previous study by Oh et al (28) demonstrated that an $\mathrm{H}_{2} \mathrm{~S}$ solution, prepared by bubbling pure $\mathrm{H}_{2} \mathrm{~S}$ gas and NaHS, dose-dependently induced HO-1 expression through the activation of the extracellular signal-regulated kinase. However, it has also been shown that increased expression of HO-1 is not necessarily accompanied by increased $\mathrm{HO}$ activity, which should also be measured (8). Therefore, HO-1 activity requires further investigation.

In conclusion, the present study demonstrated that $\mathrm{H}_{2} \mathrm{~S}$ upregulates mRNA and protein expression of HO-1 in rats with volume overload-induced CHF caused by left-to-right shunt, which may be one of the mechanisms by which $\mathrm{H}_{2} \mathrm{~S}$ attenuates volume overload-induced heart failure. However, the specific underlying mechanisms and interaction with NO require further investigation.

\section{Acknowledgements}

The study was supported by the National Natural Science Foundation of China (no. 30872787), Beijing outstanding talents training program (20081D0303200107) and Science Foundation for High-Level Medical Talents of Beijing Health System (2011).

\section{References}

1. Liu YH, Lu M, Hu LF, Wong PT, Webb GD and Bian JS Hydrogen sulfide in the mammalian cardiovascular system. Antioxid Redox Signal 17: 141-185, 2012.

2. Ji Y, Pang QF, Xu G, Wang L, Wang JK and Zeng YM: Exogenous hydrogen sulfide postconditioning protects isolated rat hearts against ischemia-reperfusion injury. Eur J Pharmacol 587: 1-7, 2008.

3. Bliksoen M, Kaljusto ML, Vaage J and Stenslokken KO: Effects of hydrogen sulphide on ischaemia-reperfusion injury and ischaemic preconditioning in the isolated, perfused rat heart. Eur J Cardiothorac Surg 34: 344-349, 2008.

4. Muellner MK, Schreier SM, Laggner H, et al: Hydrogen sulfide destroys lipid hydroperoxides in oxidized LDL. Biochem J 420 277-281, 2009

5. Johansen D, Ytrehus K and Baxter GF: Exogenous hydrogen sulfide $\left(\mathrm{H}_{2} \mathrm{~S}\right)$ protects against regional myocardial ischemia-reperfusion injury - Evidence for a role of $\mathrm{K}_{\mathrm{ATP}}$ channels. Basic Res Cardiol 101: 53-60, 2006

6. Zhu YZ, Wang ZJ, Ho P, et al: Hydrogen sulfide and its possible roles in myocardial ischemia in experimental rats. J Appl Physiol 102: 261-268, 2007.

7. Li XH, Zhang CY and Zhang T: Sodium hydrosulfide improves cardiac functions and structures in rats with chronic heart failure. Zhonghua Yi Xue Za Zhi 91: 3044-3049, 2011 (In Chinese).

8. Peterson SJ, Frishman WH and Abraham NG: Targeting heme oxygenase: therapeutic implications for diseases of the cardiovascular system. Cardiol Rev 17: 99-111, 2009.

9. Immenschuh S and Schroder H: Heme oxygenase-1 and cardiovascular disease. Histol Histopathol 21: 679-685, 2006.
10. Johnson RA, Colombari E, Columbari DS, Lavesa M, Talman WT and Nasjletti A: Role of endogenous carbon monoxide in central regulation of arterial pressure. Hypertension 30: 962-967, 1997.

11. Ndisang JF, Zhao W and Wang R: Selective regulation of blood pressure by heme oxygenase- 1 in hypertension. Hypertension 40: 315-321, 2002.

12. Otterbein LE, Zuckerbraun BS, Haga M, et al: Carbon monoxide suppresses arteriosclerotic lesions associated with chronic graft rejection and with balloon injury. Nat Med 9: 183-190, 2003.

13. Yet SF, Layne MD, Liu X, Chen YH, Ith B, Sibinga NE and Perrella MA: Absence of heme oxygenase-1 exacerbates atherosclerosis lesion formation and vascular remodeling. FASEB J 17: 1759-1761, 2003.

14. Fujimoto H, Ohno M, Ayabe S, et al: Carbon monoxide protects against cardiac ischemia - reperfusion injury in vivo via MAPK and Akt - eNOS pathways. Arterioscler Thromb Vasc Biol 24: 1848-1853, 2004

15. Liu X, Pachori AS, Ward CA, et al: Heme oxygenase-1 (HO-1) inhibits postmyocardial infarct remodeling and restores ventricular function. FASEB J 20: 207-216, 2006.

16. Ocampo C, Ingram P, Ilbawi M, Arcilla R and Gupta M: Revisiting the surgical creation of volume load by aorto-caval shunt in rats. Mol Cell Biochem 251: 139-143, 2003.

17. Yan H, Du J and Tang C: The possible role of hydrogen sulfide on the pathogenesis of spontaneous hypertension in rats. Biochem Biophys Res Commun 313: 22-27, 2004.

18. Wang X, Wang Q, Guo W and Zhu YZ: Hydrogen sulfide attenuates cardiac dysfunction in a rat model of heart failure: a mechanism through cardiac mitochondrial protection. Biosci Rep 31: 87-98, 2011.

19. Sadowski SL: Congenital cardiac disease in the newborn infant: past, present, and future. Crit Care Nurs Clin North Am 21: 37-48, 2009.

20. Ndisang JF, Tabien HE and Wang R: Carbon monoxide and hypertension. J Hypertens 22: 1057-1074, 2004.

21. Hangaishi M, Ishizaka N, Aizawa T, et al: Induction of heme oxygenase-1 can act protectively against cardiac ischemia/reperfusion in vivo. Biochem Biophys Res Commun 279: 582-588, 2000.

22. Masini E, Vannacci A, Marzocca C, et al: Heme oxygenase-1 and the ischemia-reperfusion injury in the rat heart. Exp Biol Med (Maywood) 228: 546-549, 2003.

23. Guo Y, Stein AB, Wu WJ, et al: Administration of a CO-releasing molecule at the time of reperfusion reduces infarct size in vivo. Am J Physiol Heart Circ Physiol 286: H1649-H1653, 2004.

24. L'Abbate A, Neglia D, Vecoli C, et al: Beneficial effect of heme oxygenase- 1 expression on myocardial ischemia-reperfusion involves an increase in adiponectin in mildly diabetic rats. Am J Physiol Heart Circ Physiol 293: H3532-H3541, 2007.

25. Varadi J, Lekli I, Juhasz B, et al: Beneficial effects of carbon monoxide-releasing molecules on post-ischemic myocardial recovery. Life Sci 80: 1619-1626, 2007.

26. Wang G, Hamid T, Keith RJ, et al: Cardioprotective and antiapoptotic effects of heme oxygenase-1 in the failing heart. Circulation 121: 1912-1925, 2010.

27. Qingyou Z, Junbao D, Weijin Z, Hui Y, Chaoshu T and Chunyu Z: Impact of hydrogen sulfide on carbon monoxide/heme oxygenase pathway in the pathogenesis of hypoxic pulmonary hypertension. Biochem Biophys Res Commun 317: 30-37, 2004.

28. Oh GS, Pae HO, Lee BS, et al: Hydrogen sulfide inhibits nitric oxide production and nuclear factor-kappaB via heme oxygenase-1 expression in RAW264.7 macrophages stimulated with lipopolysaccharide. Free Radic Biol Med 41: 106-119, 2006. 